\title{
Physiologically-based Modeling and Visualization of Deformable Lungs
}

\author{
Anand P Santhanam \\ University of Central \\ Florida \\ Orlando FL 32816 \\ anand@odalab.ucf.edu
}

\author{
Sumant N Pattanaik \\ University of Central \\ Florida \\ Orlando FL 32816 \\ sumant@cs.ucf.edu
}

\author{
Jannick P Rolland \\ University of Central \\ Florida \\ Orlando FL 32816 \\ jannick@odalab.ucf.edu
}

\author{
Celina Imielinska \\ Dept of Bio-infomatics \\ Columbia University \\ New York \\ Ci42@columbia.edu
}

\author{
Jack Norfleet \\ US ARMY STRICOM \\ Orlando FL 32816 \\ Jack_norfleet@peostri.army.mil
}

\begin{abstract}
A real-time physiologically-based breathing model of lungs under normal and pathological scenario has been conceived and implemented. The algorithm developed for lung deformations under various breathing scenarios uses polygonal models of lungs. The method developed avoids the "stiffness" problem observed in Mass-Spring models. Hardware acceleration of the exhalation and the inhalation process is done using vertex shaders. The method of deformation is general and can be applied to any lung model.
\end{abstract}

\section{Introduction}

Simulating breathing lungs has been one of the key issues for Medical Visualization. In order to visualize the breathing lungs of a human, it is important to superimpose a 3D lungs model over the human using Augmented Reality techniques and to simulate real time breathing. In this paper, we describe the development of a real-time physiologically-based model of breathing lungs, as well as pathologies of breathing and their 3D visualization. Specifically, we present a method and an associated algorithm for non-rigid polygonal deformations which uses an inter-node elastic interaction. We provide the linear formulation for the strain as a function of applied force using Southwell iteration. The types of deformations modeled are based on strain involved. Our algorithm is general enough for its application to any elastic object.
The organization of the paper is as follows. We review closely related work on modeling deformable models for medical visualization in Section 2. We present an overall method for physiologically-based modeling of breathing lungs in Section 3. We detail the algorithm in Section 4 and provide its implementation in Section 5. We summarize the results in Section 6.

\section{Relevant study}

The simulation of the inhalation and exhalation process of the lung has been previously modeled by Segars et al (2001) [6] using NURBS lung models for verifying the accuracy of SPECT images. The advantage of NURBS is that its deformation of a NURBS is modeled in terms of control points. The disadvantage lies in the fact that it is computationally expensive and the clique of control points for a given point remains the same, thus overshadowing the tissue properties.

A popular method for modeling discrete deformation is based on Mass-Spring models and interactions.[8] In this method an object is modeled as a collection of masses connected by springs in a structure. The Lagrangian equation of motion is used in order to model the deformation based on given set of applied forces as a function of the mass damping and stiffness. Non-linear and quasi-elastic springs can be used to model tissues such as human skin that exhibit inelastic behavior.

For modeling related to the human body, Mass-spring systems have been widely used in facial animations. A static version of Mass-Spring systems, called strain nets was used in [7]. Muscle actions or movements were represented by the application of forces to a particular region of nodes. It was later extended to another approach where a zone of influence was given to each node [10]. Assignment of spring constants, which is at the basis of movement simulation, is hand-crafted. A generic way to 
assign spring constants was developed by adjusting the spring constants in proportion to the node density [8].

The main advantage of Mass-Spring models is its simplicity. It is relatively easy to create and animate models at interactive speeds. It is also possible to use parallel computation of animation due to the local nature of the interactions between nodes. The main disadvantage of Mass-Spring models is its significant approximation of the physics involved in the dynamics of motion, which could be compensated by using a highdensity model, but this solution is computationally expensive. Furthermore, deriving values for the spring constants is a huge overhead. Most importantly, however, Mass-Spring models exhibit a problem known as "stiffness" which occurs when spring constants are large. Baraff and Watkin [1] did an analysis of the various effects of stiffness. Baraff further explained in [2] the fact that stiffness in non-rigid bodies could be compensated by formulating the non-rigid body interactions.

\section{Overview of the method for simulating breathing lungs}

The main objective of the approach is to model elastic deformations caused by an increase or a decrease in volume due to internal forces. Lungs consist of an infinite number of alveoli which takes part in gas exchange and thus the effect of individual alveoli becomes negligible. Lungs also show regional difference in expansion due to the effect of alveolis in a region.

In our method proposed, Polygonal models instead of NURBS are considered because they are more accurate, given that they are made of the exact positions of a lung rather than a set of control points. In order to deform a polygonal model, each node in the model must be scaled (i.e. displaced), therefore a scale-factor will be assigned to each point in the polygonal model. If the number of polygons is extremely large as encountered in the $3 \mathrm{D}$ models segmented from the visible human datasets, realtime rendering of software becomes challenging. However since we use Polygonal models we can have recourse to hardware rendering as further discussed in Section 5.

Given a polygonal model, we may consider the model as a wired mesh model where the force applied at any point gets transferred to its neighbors on the wired mesh based upon the strain of the neighbor. A given amount of force that is applied to any point on the surface is transferred in part to the neighbors of that point in a particular ratio. Every neighbor of that point undergoes the same force distribution process and thus every point gets an ultimate force distribution based on how well they share the force applied to them. This method of assigning strain to every point in the mesh is better than the approach associated with Mass-Spring models because it is not the exact values of the strain that matters, but it is the ratio of the strain applied on that node, to the strain of its neighbors that matters the most. Such an adaptation of a node to its neighbors avoids the "stiffness" problem observed in Mass-Spring models.

In order to specify more details of the adaptation process, we shall now turn to the physiology of the breathing process. In the normal individual in the upright position, there is a natural intra-pleural pressure gradient from the upper lung region to the lower. The negative intra-pleural pressure at the apex of the lung is normally greater than at the base. This gradient is gravity dependent and is thought to be caused by the normal weight distribution of the lungs above and below the hilum.

Because of the greater negative intra-pleural pressure in the upper lung regions, the alveoli in those regions are pre-expanded more than the alveoli in the lower regions. Therefore the compliance of the alveoli in the upper lung region is normally less than the compliance of the alveoli in the lower lung regions in a normal person in the upright position. Fig. 4 depicts on the left the percent volume intake of the alveoli as a function of their spatial location within the lung, and on the right the alveoli volume intake is plotted as a function of the intra-pleural pressure. We consider the curve shown in Fig. 4 for the initial strain assignment. We use Southwell iteration to calculate the inter-node elastic interactions. The detail of the algorithm is given in Section 4.

\section{Algorithm}

In our approach, we have pre-computed the strain at each node for a given minimum force (pressure/area) and have used the strain for scaling the model. The strain has a direction and a magnitude component. Section 4.1 and 4.2 explains the manipulation of magnitude component and Section 4.3 explains the direction component.

\subsection{Initial strain assignment}

To assign initial strain, we consider the theoretical alveoli volume intake as a function of the intra-pleural pressure. It is a sinusoidal curve with the apex having the lowest and the bottom surface having the highest values. This initial value signifies the regional change in volume of the lungs, which stems from the regional change in volume intake of alveoli.

Based on the volume intake curve of the lungs, a next step is to specify in the $3 \mathrm{D}$ model the various regions of 
the lungs that expand and contract at different rates, all interconnected based on their proximity to the most deformable region. So we will define a region of maximum deformation, compute its centroid to which a maximum strain value will be assigned. All other points are assigned initial strain values based on their proximity to the centroid. A block diagram of the assignment is shown in Fig 1.

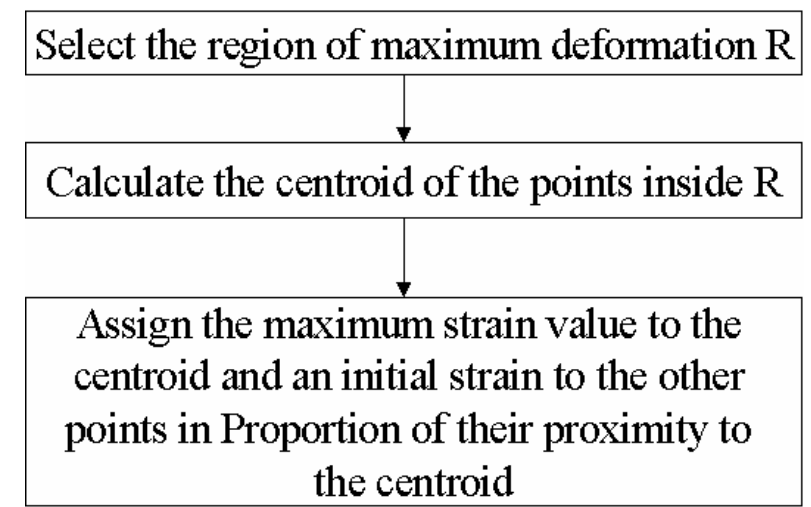

Fig. 1: Flow chart of the initial assignment value algorithm

\subsection{Iterative strain assignment}

The main aim of this step is to find out how much a node displaces when a particular known amount of force is applied on it. We start the iteration with an equal force applied on every node of the model. We then repeatedly calculate the new value of the strain as follows. Each neighbor of a node pulls its neighbor according to its own normalized strain $\mathrm{E}_{\mathrm{ij}}$ defined as

$$
E_{i j}=S_{i} / \sum S_{k}
$$

where $i$ denotes a node and $j$ its neighbor, and $k \mathbf{C}$ clique of $j$. We now define two arrays, a "Shot" array representing the final force assigned to a node, and an "Unshot" array representing the amount of force contributed by one node to its neighbors and the amount of force received by one node from its neighbors.

\section{UnShot $[i]=\min$ force \\ 2. $i=\operatorname{index}(\max (\operatorname{UnShot}))$ \\ 3.repeat until all UnShot $[i]=0$ \\ $i$. for all $j$ in the neighboghood of $i$ \\ UnShot $[j] \quad=$ UnShot $[j]+\quad E[i][j] *$ \\ UnShot[i] \\ ii. $\quad$ Shot $[i]=$ Shot $[i]+$ UnShot $[i]$ \\ iii. UnShot $[i]=0$ \\ iv. $\quad i=\operatorname{index}(\max ($ UnShot $))$}

Shot[i] is the new strain that each node is assigned to. Thus "Shot" array conveys how much each node in the model displaces for a given increase in the total volume of the model. A Schematic of the iterative strain assignment is as shown in fig 2.

\subsection{Pre-computation of the direction} component

For a given node, there exists a force from its neighbors that pulls the node towards them causing a change in direction of movement for the given node. Based on the Hooke 's law of strain, it is proportional to square of change in length between the two nodes. Given two nodes $\mathrm{A}$ and $\mathrm{B}$ which have their strain values as $a$ and $b$ respectively. $\mathrm{A}$ and $\mathrm{B}$ are scaled from a point $\mathrm{O}$ by their strain values. The increase in distance between $A$ and $B$ is equal to $\left(a^{*} \mathrm{n}_{\mathrm{A}}-b^{*} \mathrm{n}_{\mathrm{B}}\right)$, where $\mathrm{n}_{\mathrm{A}}$ and $\mathrm{n}_{\mathrm{B}}$ are the normals of $\mathrm{A}$ and $\mathrm{B}$ from $\mathrm{O}$ respectively. The final direction is computed as the average of change in direction induced by all the neighbors.

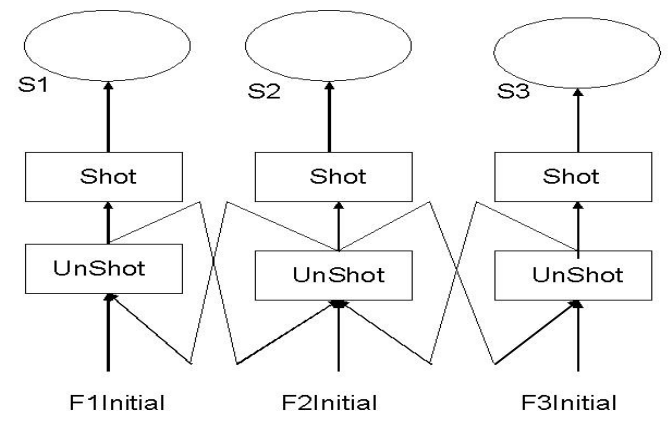

Fig. 2 Iterative algorithm

Given node $i$

For all $j$ where $j \in$ clique of $i$.

For all $\boldsymbol{l}$ where $k \in$ clique of $i$ except $j$

N1[ l] = Shot [i]*Normal[i] -

Shot [l]*Normal[l]

Sum $=\sum|N 1[l]|^{2}$

$D j=\mid($ Shot $[i] *$ Normal $[i]-$ Shot $[j] *$ Normal $[j]) \mid$

$N / j l=\left(\operatorname{Sum} /(D j)^{2}\right) *$ Normal $[j]$

Normal[ $i]=$ Normalize $\left(\sum N[k]\right)$ where $k \in$ clique of

\section{Implementation}

\subsection{Lung dynamics simulation}

We have used two lungs models to implement this algorithm with one being a low point-density polygonal mesh (255 vertices) model and the other being a very high point- density model (3M points) obtained from the Bio-Medical Department of Columbia University. We have given the outputs that show continuous expansion and contraction for both the models. The resulting images have been shown in the Figures 6, 7 and 8.

\subsection{Initial Strain Assignment}

The centroid of the points are assigned the maximum strain and other points are assigned strain as a function their Euclidean distance from the centroid. This function 
is shown in the Fig 4. We have a Sigmoid curve which could be split into an exponential curve until the mid point and then a gaussian curve. The initial assignments are given in table 3 .

Table. 3 Initial assignment table

\begin{tabular}{|l|l|}
\hline $\begin{array}{l}\text { Initial Strain } \\
\text { Assigned }\end{array}$ & $\begin{array}{l}\text { Ratio of } \\
\text { distance between } \\
\text { the point and } \\
\text { centroid with } \\
\text { respect to the } \\
\text { farthest point and } \\
\text { the centroid }\end{array}$ \\
\hline 0.4 & 0 \\
\hline 0.2 & 0.5 \\
\hline 0.075 & 1 \\
\hline
\end{tabular}

\subsection{Scaling nodes of the models}

The scaling of every node is done from the centroid of all the points as follows: We define SAV as the surface area for a given volume of lungs. We denote $j$ as the required increase in volume.

\section{During every Inhalation step: Position $(i)=1+(\operatorname{Shot}[i] * S A V(j)) *$ Normal $[i]$ \\ During every Exhalation step: Position $(i)=1-(\operatorname{Shot}[i] * S A V(j)) * N o r m a l[i]$} (2)

(3)

Where Position(i) is the position (x, y, z) of the node $i$. With this model we can simulate patho-physical conditions Any physical condition could be explained interms of changes in initial assignment curve and changes in the inhalation and the exhalation step equations.

\subsection{Assessment of assignment of strain values}

How the proposed method is parallel to method by segars and how it differs is now discussed.In our proposed method we model the internal changes, that takes place and in the method by Segars they model the external changes that makes the lung deform. Since the interpleural cavity is vacuum both the internal changes and external forces are closely bonded. The strain factor for every node in our proposed method could be equated to the weight assigned to a control point in the NURBS model. In both the models a set of points that form the neighborhood calculate the final position.In the method proposed, computation overload in rendering, increases less with increase in density of points as compared to NURBS. Scaling a point in the proposed method takes approximately 3 arithmetic operations. Moving a point in 4D NURB surface takes approximately 81 arithmetic operations.

\subsection{Hardware rendering}

For real-time simulation of the breathing using high-density polygonal model of the lungs, we implemented the algorithm in programmable NVIDIA GeForce 4 using CG2.0. All the vertices of the lungs model were first loaded into the AGP memory. Using the current implementation we are able to get an interactive frame rates for any given deformation using the highdensity lungs model.

\section{Conclusion}

We have presented a new algorithm for real-time rendering of normal and pathological breathing lungs that can be visualized in 3D in an augmented reality (Fig3) application. This method has proven to be a useful tool for deformation of objects affected by internal forces. The future question remains as to how this can be extended for with open models and other deformation models and how pathologies could be modeled.

\section{Acknowledgement}

This research was supported by the US Army Simulation, Training, and Instrumentation Command (STRICOM) for the real-time visualization of deformable lung models using Augmented Reality Environment developed by ODALab .(http://odalab.creol.ucf.edu)

\section{References}

[1] D. Baraff and Witkin .A. Dynamic Simulation of nonpenetrating flexible bodies. ACM Siggraph 1992.

[2] D. Baraff . Analytical methods for dynamic Simulation of non-penetrating rigid bodies. ACM Siggraph 1989

[3] Davis L. J. Rolland, F.Hamza-Lup, Y.Ha, J.Norfleet, B.Pettitt, C.Imielinska, Alice's Adventures of Wonderland: A unique technology Enabling a Continuum of Virtual Environment Experience, Computer Graphics and Applications February 2003

[4] T.D.Jardins. Pulmonary anatomy and Physionlogy. ITP publications 1998.

[5] L. Neumann, Neumann .A. Radiosity and hybrid methods. ACM Transactions 1995

[6] W.Paul Segars, David S Lalush and Benjamin M W Tsui. Modeling Respiratory mechanics in the MCAT and the splinebased MCAT systems. IEEE Trans Nucl Sci, 48(1): 89-97, 2001

[7] S.Platt and N.Baldar. Animating facial expressions. Computer Graphics 1981

[8] Sarah F F Gibson and Brian Mirtich. A survey of Deformable modeling in Computer Graphics. November 1997 MERL. 
[9] D.Terzopolous and K.Waters. Physically based facial modeling analysis and animation. Journal of Visualization and Computer Animation 1990.

[10] Waters. A muscle model for animating three-dimensional facial expression. ACM Siggraph 87

[11] J West. Physiological modeling of Respiratory mechanics Addison Wesley publications

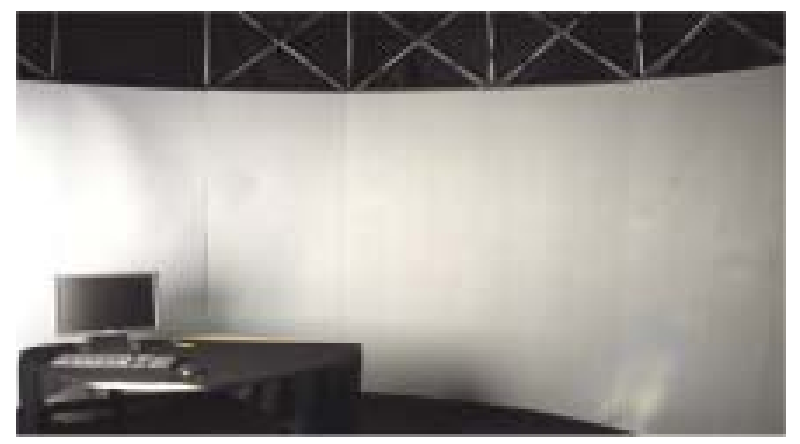

Fig. 4: A fifteen feet diameter ARC display

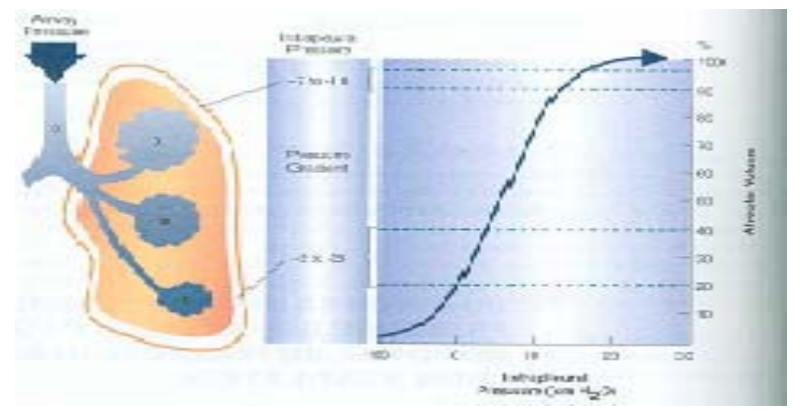

Fig. 5: Alveoli volume intake as a function of intra-pleural pressure in upright position (Courtesy: Cardiopulmonary Anatomy and Physiology by Terry Des Jardins).

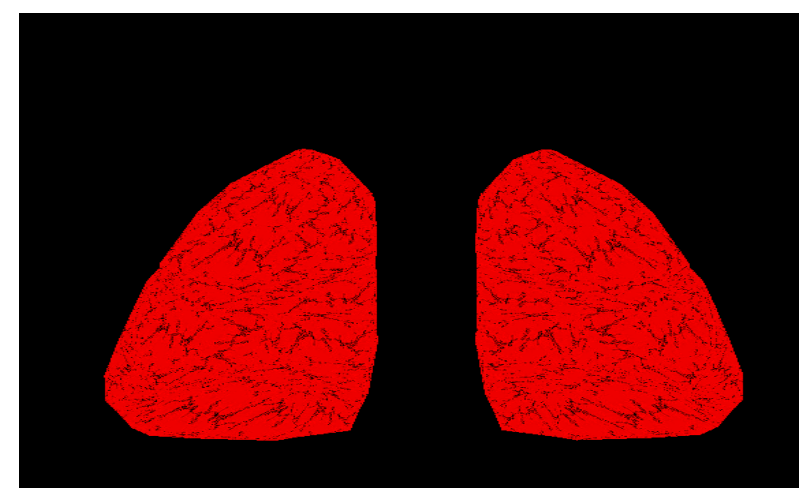

Fig 6: The initial shape of the lung

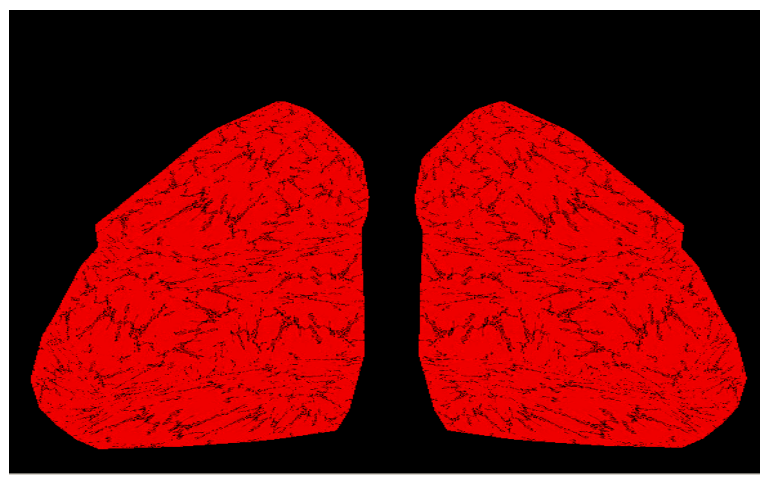

Fig. 7: The shape of the lung in expansion

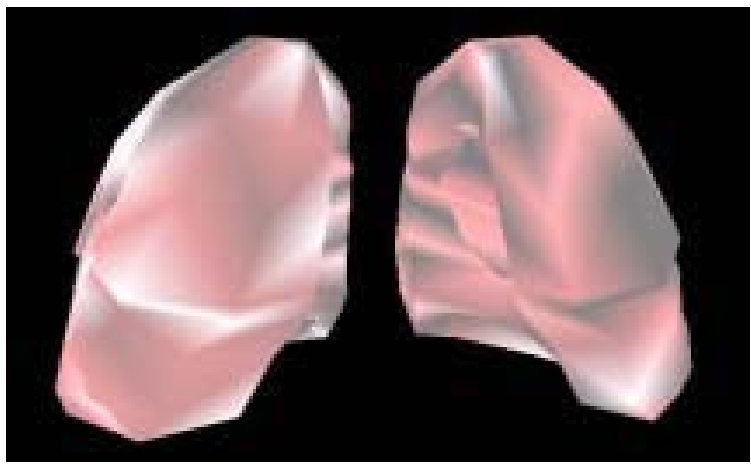

Fig. 8: A 3D view of the breathing lungs with smooth shading and lighting.

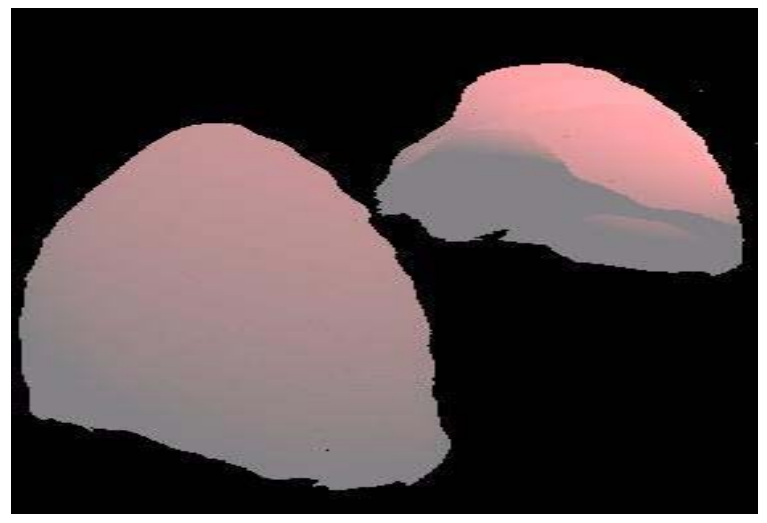

Fig. 9: A 3D model of lungs obtained from department of Biomedical Engineering, Columbia University for Visible Human Simulator. 